\title{
Stabilization of the promoter nucleosomes in nucleosome-free regions by the yeast Cyc8-Tupl corepressor
}

\author{
Kaifu Chen, ${ }^{1,2,8}$ Marenda A. Wilson, ${ }^{3,4,5,8}$ Calley Hirsch, ${ }^{3,4,5}$ Anjanette Watson, ${ }^{6}$ \\ Shoudan Liang, ${ }^{4,7}$ Yue Lu, ${ }^{4,5}$ Wei Li, ${ }^{1,2,9}$ and Sharon Y.R. Dent ${ }^{3,4,5,9}$ \\ ${ }^{1}$ Division of Biostatistics, Dan L. Duncan Cancer Center, Baylor College of Medicine, Houston, Texas 77030, USA; ${ }^{2}$ Department \\ of Molecular and Cellular Biology, Baylor College of Medicine, Houston, Texas 77030, USA; ${ }^{3}$ Program in Genes and Development, \\ The University of Texas MD Anderson Cancer Center, Houston, Texas 77030, USA; ${ }^{4}$ Center for Cancer Epigenetics, The University \\ of Texas MD Anderson Cancer Center, Houston, Texas 77030, USA; ${ }^{5}$ Department of Molecular Carcinogenesis, The University of Texas \\ MD Anderson Cancer Center, Houston, Texas 77030, USA; ${ }^{6}$ University of Vermont Bioinformatics Core, Burlington, Vermont 05405, \\ USA; ${ }^{7}$ Department of Bioinformatics and Computational Biology, The University of Texas MD Anderson Cancer Center, Houston, \\ Texas 77030, USA
}

\begin{abstract}
The yeast Cyc8 (also known as Ssn6)-Tupl complex regulates gene expression through a variety of mechanisms, including positioning of nucleosomes over promoters of some target genes to limit accessibility to the transcription machinery. To further define the functions of Cyc8-Tupl in gene regulation and chromatin remodeling, we performed genome-wide profiling of changes in nucleosome organization and gene expression that occur upon loss of CYC8 or TUPI and observed extensive nucleosome alterations in both promoters and gene bodies of derepressed genes. Our improved nucleosome profiling and analysis approaches revealed low-occupancy promoter nucleosomes (P nucleosomes) at locations previously defined as nucleosome-free regions. In the absence of $C Y C 8$ or TUP1, this P nucleosome is frequently lost, whereas nucleosomes are gained at -1 and +1 positions, accompanying up-regulation of downstream genes. Our analysis of public ChIPseq data revealed that $\mathrm{Cyc} 8$ and Tupl preferentially bind TATA-containing promoters, which are also enriched in genes derepressed upon loss of $C Y C 8$ or TUP1. These results suggest that stabilization of the P nucleosome on TATA-containing promoters may be a central feature of the repressive chromatin architecture created by the Cyc8-Tupl corepressor, and that releasing the $\mathrm{P}$ nucleosome contributes to gene activation.
\end{abstract}

[Supplemental material is available for this article.]

Corepressor complexes do not interact directly with DNA but are brought to target gene promoters through interactions with sequence-specific DNA binding repressors (Payankaulam et al. 2010). The yeast Cyc8-Tup1 complex was the first transcriptional corepressor to be defined (Keleher et al. 1992; Cooper et al. 1994). Subsequent identification of corepressors in other organisms, such as the proteins CTBP1 (Chinnadurai 2003), NCOR2 (Heinzel et al. 1997; Nagy et al. 1997), NRIP1 (Rosell et al. 2011), and SIN3A (Silverstein and Ekwall 2005) in mammals, and Groucho (Buscarlet and Stifani 2007) in Drosophila demonstrated that corepressor functions are essential for normal cell growth, response to environmental signals, and developmental cues.

Many corepressors function through the organization of chromatin into a structure that is not conducive to transcription. Most are directly involved in the recruitment of histone modification enzymes such as histone deacetylases (HDACs) (Zamir et al. 1997; Burke and Baniahmad 2000; Li et al. 2000; Davie et al. 2003; Perissi et al. 2010), histone methyltransferases (Shi et al. 2003), or histone demethylases (Shi et al. 2003) to target gene promoters.

\footnotetext{
${ }^{8}$ These authors contributed equally to this work.

${ }^{9}$ Corresponding authors

E-mail sroth@mdanderson.org

E-mail WL1@bcm.edu

Article published online before print. Article, supplemental material, and publication date are at http://www.genome.org/cgi/doi/10.1101/gr.141952.112.
}

Cyc8-Tup1 recruits HDACs to target promoters, and Tup1 directly interacts with under-acetylated histones $\mathrm{H} 3$ and $\mathrm{H} 4$ to stabilize association of the corepressor with the repressed gene in a feedforward regulatory loop (Watson et al. 2000). The Cyc8-Tup1 complex can also act independently of chromatin alterations to repress transcription through negative interactions with components of the RNA polymerase II holoenzyme (Conlan et al. 1999; Smith and Johnson 2000; Papamichos-Chronakis et al. 2002). However, the mechanism of this inhibition is not well understood. Previous studies of the effects of the Cyc8-Tup1 complex on chromatin structure have been limited to a few target genes. These studies revealed a variety of effects of the corepressor on chromatin architectures. In some instances, Cyc8-Tup1 influences nucleosome locations only in the promoter region, as is the case at RNR2 and RNR3 (Li and Reese 2001; Davie et al. 2002). At other genes, such as STE2 and STE6, Cyc8-Tup1 creates an array of highly positioned nucleosomes that extends from the promoter into the gene body (Ducker and Simpson 2000; Davie et al. 2002). At the SUC2 locus, recruitment of Cyc8-Tup1 causes even longer-range chromatin reorganization, extending from upstream of the promoter region into intergenic regions (Fleming and Pennings 2007). These studies suggest that Cyc8-Tup1 may mediate chromatin organization and gene repression via a variety of mechanisms.

To better define how these factors influence transcription patterns and chromatin organization and to determine whether or not a "core chromatin alteration" might be universally associated 
with Cyc8-Tup1-mediated repression, we carried out genome-wide nucleosome mapping and gene expression studies in wild-type cells and in cells lacking either CYC8 or TUP1. Our data confirm that the functions of these proteins significantly overlap, but also reveal that loss of TUP1 causes a greater number of chromatin alterations than does loss of $C Y C 8$. We also find evidence of a promoter-associated nucleosome in what is typically thought of as a nucleosome-free region (NFR). Stabilization of this nucleosome appears to be a central role of Cyc8-Tup1 complex in transcription repression.

\section{Results}

\section{An overview of the high-quality nucleosome data}

To define genome-wide changes in chromatin caused by loss of CYC8 or TUP1, nucleosomes were prepared from isogenic wildtype (BY4742), cyc8s, and tup1s cells after varying degrees of micrococcal nuclease (MNase) digestion, followed by isolation of mononucleosomal DNA and sequencing. Three replicates of each strain (nine samples) were subjected to Illumina sequencing, yielding a total of 464 million reads (Table 1$)$. About half $(50.73 \%)$ of the reads passed quality filtering and could be uniquely mapped to the yeast genome. Visual inspection of individual genomic regions revealed that the data obtained were in general highly reproducible between replicates of the same strain (Supplemental Fig. 1); no reads were mapped to the open reading frame (ORF) of $C Y C 8$ in the $C y c 8 \Delta$ strain or TUP1 in the tup $1 \Delta$ strain, respectively; however, corresponding reads mapped to the flanking regions appear reproducible among replicates, suggesting that the knockouts were correctly made and had no adverse effects on the surrounding regions.

Previous work revealed that there are about 50,000-70,000 nucleosomes in the yeast genome (Jiang and Pugh 2009); each nucleosome has an "occupancy level," which refers to the frequency at which the nucleosome is present in a given cell population (Fig. 1A). We identified a total of 66,167 nucleosomes by pooling all nine samples. A similar number of nucleosomes could also be identified from each individual sample (Table 1). Pairwise Pearson correlation analysis indicates that nucleosome occupancies between replicates are highly reproducible, with correlation coefficients all higher than 0.90 (Fig. 1C); we also observed significant overlap of high-occupancy nucleosomes between replicates with Fisher's exact $P$-values lower than $1 \times 10^{-300}$ (Fig. 1C).

Although the nucleosome data were highly reproducible, some differences between replicates could be observed. We were interested in whether the nucleosome changes between the different strains were larger than those between replicates of the same strain. Therefore, the top $5 \%$ of nucleosomes with the highest

Table 1. A summary of the data sets generated in this work

\begin{tabular}{lcccc}
\hline $\begin{array}{l}\text { Cell } \\
\text { type }\end{array}$ & Replicates & $\begin{array}{c}\text { Reads } \\
\text { count }\end{array}$ & $\begin{array}{c}\text { High quality } \\
\text { and unique (\%) }\end{array}$ & $\begin{array}{c}\text { Nucleosome } \\
\text { count }\end{array}$ \\
\hline \multirow{2}{*}{ Wild-type } & $\# 1$ & $43,299,462$ & 47.95 & 66,247 \\
& $\# 2$ & $38,648,096$ & 53.83 & 61,831 \\
\multirow{4}{*}{ tup14 } & $\# 3$ & $37,061,628$ & 43.12 & 62,445 \\
& $\# 1$ & $38,492,694$ & 39.88 & 67,024 \\
& $\# 2$ & $75,843,736$ & 46.02 & 65,530 \\
\multirow{2}{*}{ cyc84 } & $\# 3$ & $56,064,944$ & 49.01 & 67,280 \\
& $\# 1$ & $58,023,598$ & 64.45 & 65,090 \\
\multirow{3}{*}{ All } & $\# 2$ & $46,975,834$ & 43.19 & 64,684 \\
& \#3 & $70,231,954$ & 61.52 & 65,202 \\
& - & $464,641,946$ & 50.73 & 66,167 \\
\hline
\end{tabular}

standard deviation among all nine samples were selected for hierarchical clustering analysis (Fig. 1D). The results indicate that replicates from the same strain always clustered together. Interestingly, $c y c 8 \Delta$ strains clustered closer to wild-type strains, whereas tup1d strains seem to be outliers, suggesting that loss of TUP1 causes more changes at the nucleosome level than does loss of $C Y C 8$.

\section{Discovery of the P nucleosome}

Previous reports indicate that nucleosome positions have a robust pattern relative to gene structure. When nucleosome density is plotted as a function of distance relative to the transcription start site (TSS), a -1 nucleosome is positioned close to TSS from $-307 \mathrm{bp}$ to $-111 \mathrm{bp}$, and $\mathrm{a}+1$ nucleosome is positioned from $-5 \mathrm{bp}$ to +144 bp (Jiang and Pugh 2009). Following the +1 nucleosome, multiple nucleosomes are well positioned along the gene body; a 100-bp region between the $-111 \mathrm{bp}$ and $-5 \mathrm{bp}$ is free of nucleosomes and is referred to as the NFR (Jiang and Pugh 2009). We observed similar patterns in nucleosome occupancy flanking the TSS, confirming the quality of our data analyses (Fig. 1E).

However, when grouping nucleosomes relative to a common genomic feature such as TSS, a small group of nucleosomes (low nucleosome count) could have a high average occupancy, whereas a large group of nucleosomes (high nucleosome count) could have a low average occupancy (Fig. 1B). Under these conditions, lowoccupancy nucleosomes would be hard to observe based on occupancy analysis. Thus we plotted the nucleosome count instead of occupancy flanking TSS, and as a result observed an additional nucleosome between the -1 and +1 nucleosomes (Fig. 1E). Because this nucleosome is located in the canonical NFR in the promoter, we defined it as the P nucleosome in this study. Interestingly, the $\mathrm{P}$ nucleosome was more distinguishable from the neighboring -1 and +1 nucleosomes in wild-type cells, whereas it is less enriched in the absence of CYC 8 or TUP1. Conversely, the -1 and +1 nucleosomes are more enriched in the $c y c 8 \Delta$ and tup $1 \Delta$ strains relative to the wild-type strain (Supplemental Fig. 2).

To further test whether a lower occupancy level is the reason that this nucleosome was not reported previously, we separately plotted the count of high-occupancy and low-occupancy nucleosomes flanking TSS (Fig. 1F). The high-occupancy group showed the canonical NFR as reported previously, with no evidence of the P nucleosome. In contrast, the P nucleosome can be seen clearly in the low-occupancy group and is even more abundant than the -1 nucleosome. Another potential reason that the P nucleosome has not been reported previously might be MNase overdigestion. Most previous nucleosome data were derived from fully MNase-digested samples, resulting in loss of the P nucleosome in canonical NFR (Xi et al. 2011). To test this possibility, we analyzed public nucleosome data generated using complete digestion or partial digestion conditions (Xi et al. 2011). As expected, data from completely digested samples show a canonical NFR with no evidence of a P nucleosome, whereas the $\mathrm{P}$ nucleosome could be easily observed in the data from partially digested samples (Supplemental Fig. 3). Our mononucleosomal DNA was isolated from an array of digestion conditions, which made it easier to identify the P nucleosomes in our analyses.

\section{Cyc8 and Tupl show both common and unique effects on nucleosome occupancy across the genome}

Previous lower-resolution mapping studies suggested that the Cyc8-Tup1 complex represses transcription by locking nucleo- 
A Nucleosome occupancy of individual nucleosome in a cell population

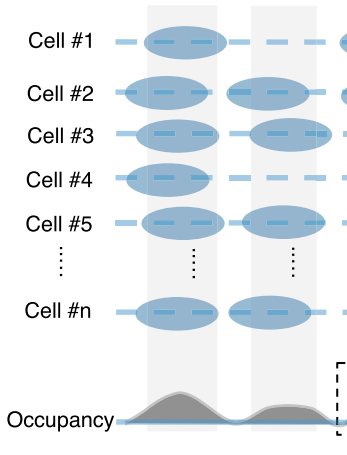

Nucleosomes ।

II

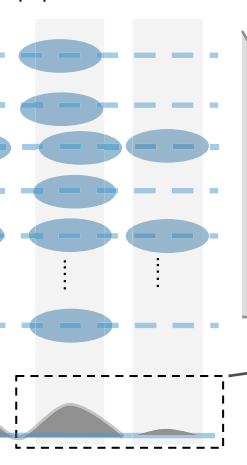

III

IV

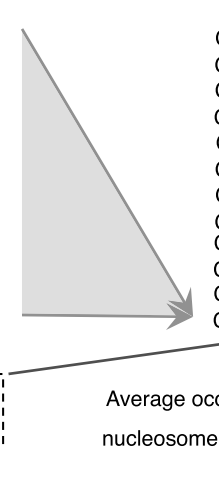

nucleosome count
C

Position Overlap of high-occupancy nucleosomes

Pearson correlation of nucleosome occupancy
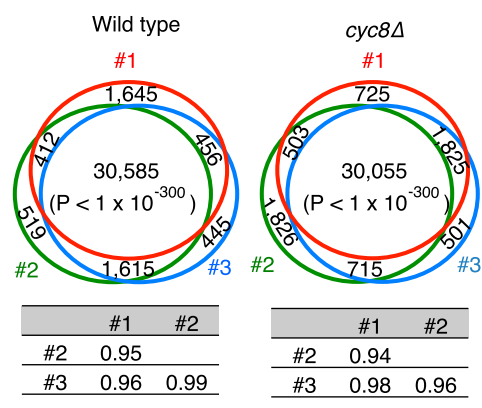

B Average nucleosome count and occupancy flanking gene body of a group of genes

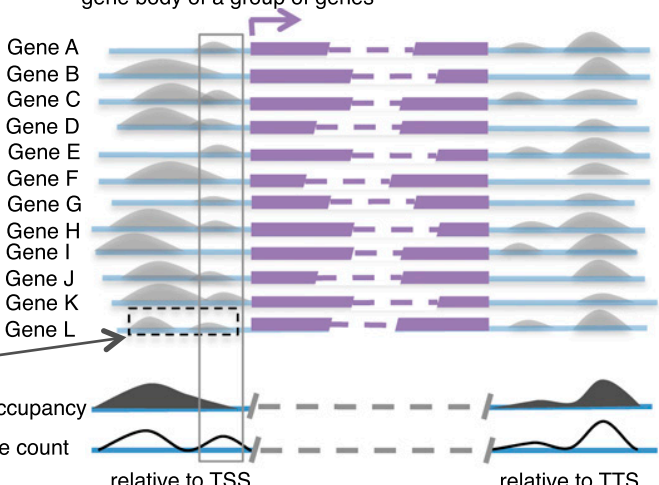

relative to TSS
E

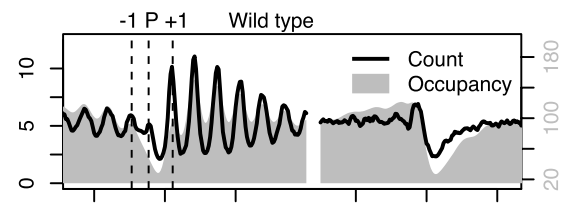

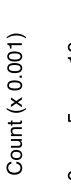
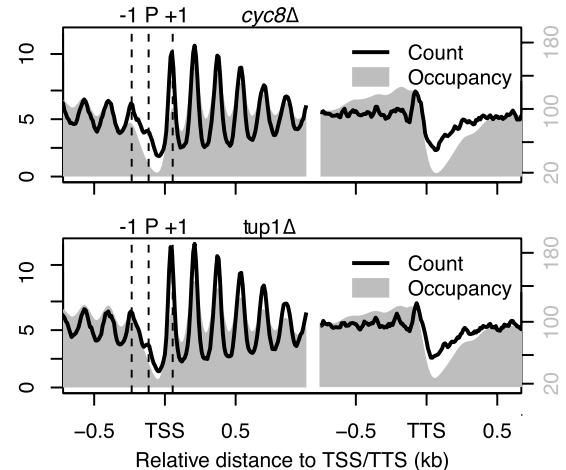

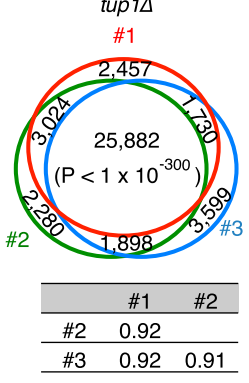

○

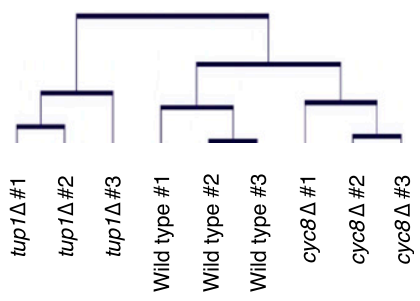

$\mathbf{F}$

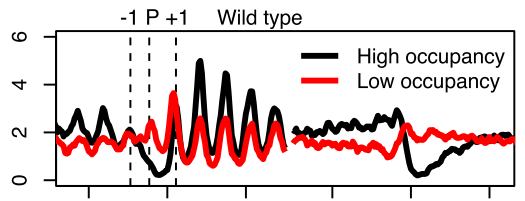

$-1 \mathrm{P}+1 \quad c y c 8 \Delta$
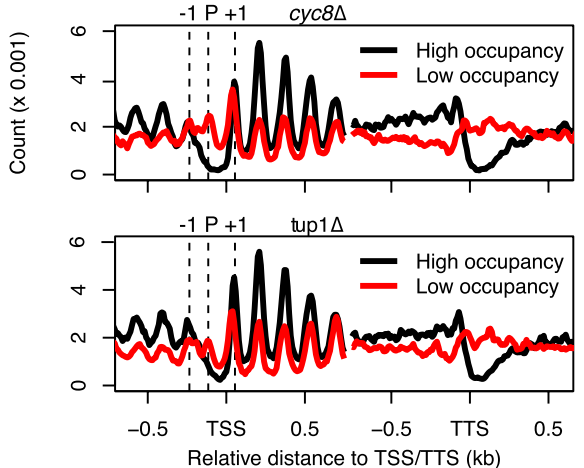

Figure 1. Nucleosome position and occupancy analysis based on high-quality maps enable observation of a $\mathrm{P}$ nucleosome on promoters. $(A) \mathrm{A}$ cartoon to show nucleosome position and occupancy analysis at a single genomic locus in a cell population. Theoretically, nucleosomes (blue ovals) may be located anywhere on a DNA fragment (blue solid line); "nucleosome occupancy" refers to the frequency at which nucleosomes present at a specific position. Technically, nucleosome occupancy can be measured by local sequencing depth (gray area under curve) at each nucleotide across the genome, and nucleosomes (gray bars) can be defined as occupancy peaks whose sizes are close to a nucleosome unit length. (Blue dash lines) Copies of the same DNA fragment in different cells as labeled on left. (B) A cartoon to show nucleosome position and occupancy analysis relative to a putative gene group. When grouping nucleosomes based on a common genomic feature such as a transcription start site (TSS) or transcription terminal site (TTS), a group of nucleosomes (gray solid box) with high nucleosome count (solid curve) might not be observable due to lower average occupancy (black area under curve) relative to neighboring nucleosomes. (Purple bars and dashed lines) Different genes (labeled on left); (purple arrow) the transcription direction and TSS. (Blue solid lines) Genomic regions flanking a gene group. (Black dash boxes in $A$ and $B$ ) The same putative genomic locus. (C) Venn diagrams representing the overlap of high-occupancy nucleosomes between replicates, with the Pearson correlation coefficients of nucleosome occupancy between replicates shown below. All nucleosomes are used for calculation of Pearson correlation coefficients, whereas the $50 \%$ nucleosomes ranked by occupancy were retrieved for estimation of position overlap, with the overlapping $P$-value estimated based on Fisher's exact test. $(D)$ Hierarchical clustering of all nine samples based on nucleosome occupancy. The top 5\% of nucleosomes ranked by standard deviation of occupancies among the nine samples were used in the unbiased clustering. (E) Average nucleosome occupancy and count plotted as a function of distance to the TSS or TTS. $(F)$ Average nucleosome count of high- and low-occupancy nucleosomes plotted as a function of distance to the TSS or TTS.

\section{Genome Research}

www.genome.org 
somes on or near the promoters of target genes (Roth et al. 1990; Cooper et al. 1994). If so, then the removal of this complex should result in nucleosome loss at Cyc8-Tup1 gene targets, consistent with the loss of the P nucleosome in the $c y c 8 \Delta$ and tup1 $1 \Delta$ strains described above. To further address nucleosome changes related to Cyc8-Tup1 functions, we examined nucleosome gain or loss events at previously defined Cyc8-Tup1 target genes (Fig. 2A). In general, nucleosome changes appear very reproducible between replicates; nucleosome loss events tend to happen on promoter proximal regions, whereas nucleosome gain was observed in both promoters and gene bodies (Fig. 2A; data not shown). The HXT genes, which are hexose transporters that function in the transport of glucose across the cell membrane, are repressed by Cyc8-Tup1 in the presence of glucose (Ozcan and Johnston 1995; Kim et al. 2003). The FLO genes, which regulate yeast flocculation, are repressed by the Cyc8-Tup1 complex under normal growth conditions (Fleming and Pennings 2001); on the promoters of these genes and others, we observed an extensive loss of nucleosomes in both $c y c 8 \Delta$ and tup $1 \Delta$ strains relative to wild-type cells. However, at other locations including known Cyc8-Tup1 target genes such as STE2 (Cooper et al. 1994) and RNR3 (Li and Reese 2001), nucleosome rearrangement appeared to be more complicated; the major nucleosome alteration for STE2 is a shift of the +1 nucleosome toward the $5^{\prime}$ direction. The -1 nucleosome also showed some location shift in the same direction, whereas the -2 nucleosome remained stable. For RNR3, nucleosomes became fuzzier at the $5^{\prime}$ end of the gene body in the $c y c 8 \Delta$ and tup $1 \Delta$ strains. This change in fuzziness appeared to reflect a nucleosome occupancy decrease at wild-type dyads and increased occupancy in wild-type linker regions.

Our analyses indicate that there are approximately fivefold more nucleosome changes (nucleosome loss and gain) in tup1s strains than in $c y c 8 \Delta$ strains (Fig. 2B), suggesting that Tup1 has greater effects on nucleosome organization. This observation agrees with our unbiased clustering results based on the top 5\% high standard deviation nucleosomes (Fig. 1D). Since Cyc8 and Tup1 have been characterized to work together as a corepressor complex, we asked whether the genomic locations of nucleosome

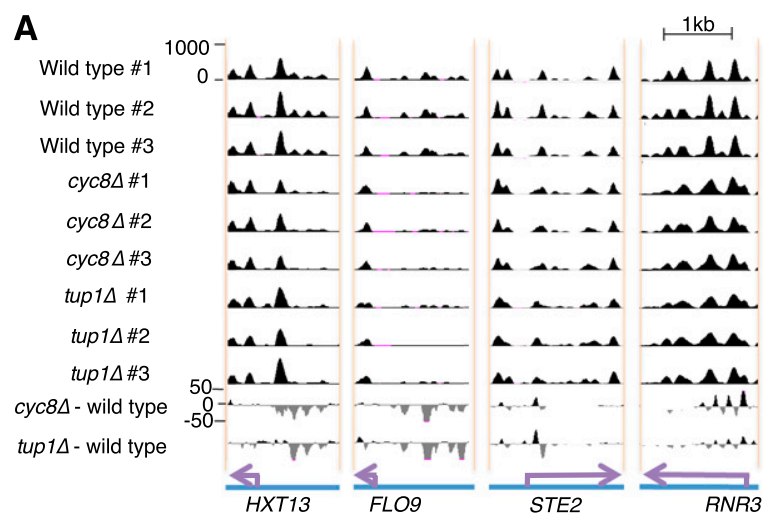

C

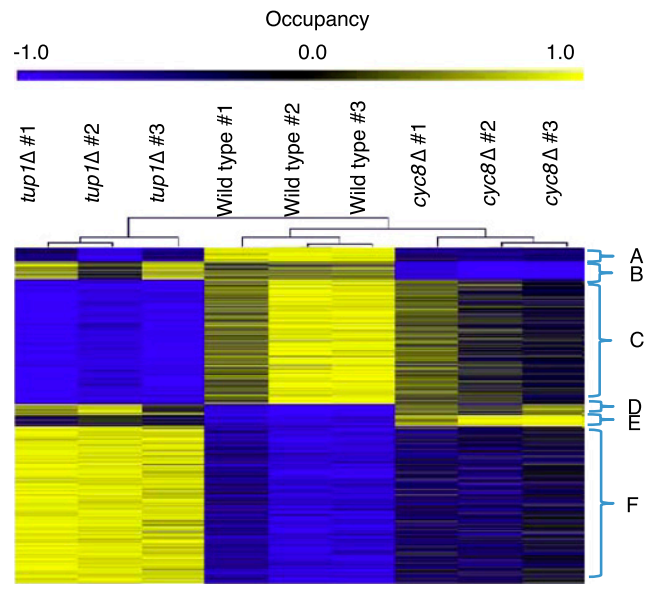

D
B

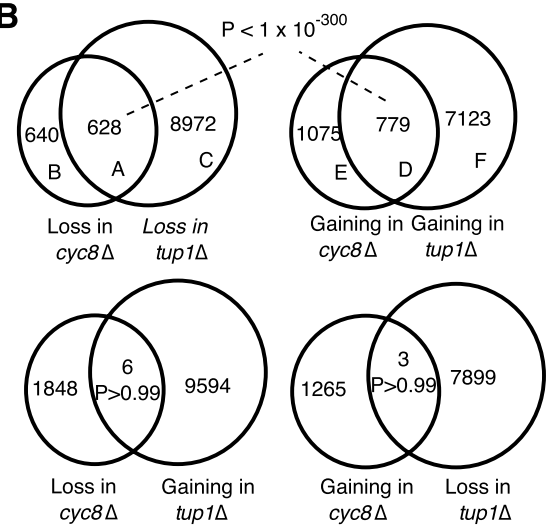

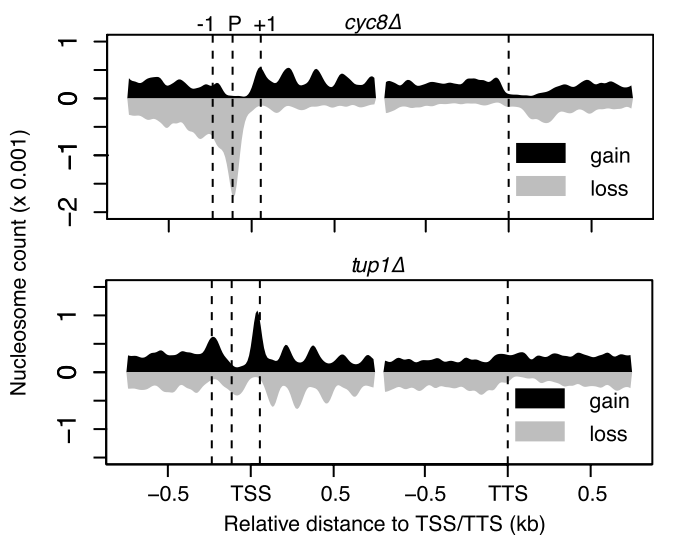

Figure 2. Cyc8 and Tup1 show common and individual global effects on nucleosome positioning. (A) Snapshots of the nucleosome maps on some known target genes of the Cyc8-Tup1 complex. The gene locus (purple arrows) is labeled at the bottom. Each of the top nine tracks represents nucleosome occupancy (black area) in one sample, with the sample name labeled at left. The cyc8s-Wild-type and tup1 $\Delta$-Wild-type tracks show the occupancy differences between the corresponding strains, with nucleosome gain (black; positive) or nucleosome loss (gray; negative) plotted separately. (B) Venn diagrams showing the overlapping nucleosome gain and loss events of $c y c 8 \Delta$ and tup $1 \Delta$ strains. The overlap $P$-value is estimated based on a Fisher's exact test. (C) A heat map to show occupancy of each nucleosome in each sample. Each row represents a nucleosome, and each column represents a sample. Only nucleosomes showing altered occupancy in cyc8s or tup $1 \Delta$ strains were used in the plot. Samples were hierarchically clustered, and for each nucleosome, the occupancy values from all nine samples were scaled to a range between -1 and 1 . Nucleosomes were grouped and labeled based on the Venn diagrams in $B$. (D) Count of nucleosome gain or loss events plotted as a function of distance to the TSS or TTS. 
changes in $c y c 8 \Delta$ and tup1s strains overlap. As expected, the overlap between these strains was highly statistically significant with a Fisher's exact $P$-value $<1 \times 10^{-300}$; as a control, sites of nucleosome loss in $c y c 8 \Delta$ strains showed little overlap with sites of nucleosome gain in the tup $1 \Delta$ strains, and vice versa, with a Fisher's exact $P$-value $>0.99$ (Fig. $2 \mathrm{~B}$ ). A quantitative analysis of nucleosome changes in the two mutant strains in a high-resolution nucleosome occupancy heat map further confirmed a significant overlap (Fig. 2C); a significant loss of nucleosomes in one mutant strain relative to wild-type cells was often mirrored in the second mutant strain (Fig. 2B,C, groups A, B, and C); a similar trend could also be observed for a gain in nucleosomes (Fig. 2B,C, groups D, E, and F). Taken together, these data confirm that Tup1 and Cyc8 work together in chromatin organization at many genes.

To further investigate the roles of Cyc8 and Tup1 in nucleosome organization, we plotted the count of the nucleosome gain and loss events separately relative to gene structure (Fig. 2D). In the cyc8s strain, the loss of nucleosomes (groups A and $\mathrm{B}$ ) is enriched on the promoters but is close to or lower than background levels on the gene body. In the tup1s strain, nucleosome loss (groups A and C) is observed on promoters as well as the $5^{\prime}$ end of gene bodies. Nucleosome gain (groups D and E, or groups D and F) events are enriched on gene bodies in both the $c y c 8 \Delta$ and tup $1 \Delta$ strains. No big difference was observed in analysis of genes affected uniquely by Cyc8 or Tup1 (groups B and E for $c y c 8 \Delta$, or C and F for tup1 $\Delta$ ) or in analysis of genes affected by both Cyc8 and Tup1 for the plot (Supplemental Fig. 4).

\section{Cyc8 and Tupl show common and independent effects on transcriptional regulation}

To investigate the effects of Cyc8 and Tup1 on transcription, we profiled gene expression patterns of $c y c 8 \Delta$, tup1s, and wild-type cells by microarray. Based on a $Q$-value cutoff of 0.05 and a fold change cutoff of 1.5, 509 and 465 genes were up-regulated and down-regulated in $c y c 8 \Delta$ strains relative to wild-type strains, respectively. Similarly, differentially expressed genes were detected in tup1s strains, with 564 and 620 genes up-regulated and downregulated (Fig. 3A). Regulated genes showed a significant overlap between $c y c 8 \Delta$ and tup $1 \Delta$ strains, with 365 common up-regulated genes $\left(P\right.$-value $\left.=1 \times 10^{-692}\right)$, and 210 common down-regulated genes $\left(P\right.$-value $\left.=1 \times 10^{-204}\right)$; as a control, little overlap was observed between up-regulated genes in $c y c 8 \Delta$ strains and downregulated genes in tup1s strains ( $P$-value $>0.99)$. An expression heat map also confirmed an overlap in regulated genes, because increased expression in one mutant strain was often mirrored in the second mutant strain (Fig. 3A,B, groups A, B, and C). A similar trend was also observed for down-regulated genes (Fig. 3A,B, groups D, E, and F). Genes in each one of these categories (regulated by both Cyc8 and Tup1, by Tup1 alone, or by Cyc8 alone) were validated by qRT-PCR (Supplemental Fig. 5.) The strains used in our studies were created in a BY4742/MAT $\alpha$ background, which allowed us to follow gene expression levels of internal controls, such as the a-cell-specific transcripts STE2 that are negatively regulated by Cyc8-Tup1; we also compared gene expression levels of the known Cyc8-Tup1 target FLO genes, HXT genes, and the DNA damage-induced gene RNR3 in each strain (Fig. 3C). As previously reported (Ozcan and Johnston 1995; Fleming and Pennings 2001; Kim et al. 2003), all of these genes were up-regulated in the absence of either CYC8 or TUP1.

The overlap of up-regulated genes between $c y c 8 \Delta$ and tup $1 \Delta$ strains appeared more significant than that for down-regulated
A
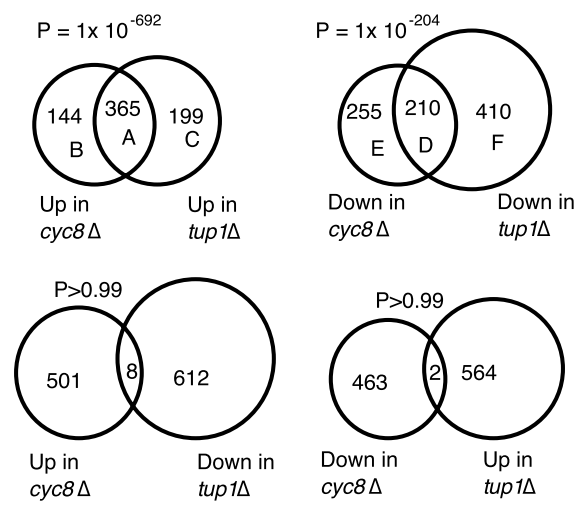

B
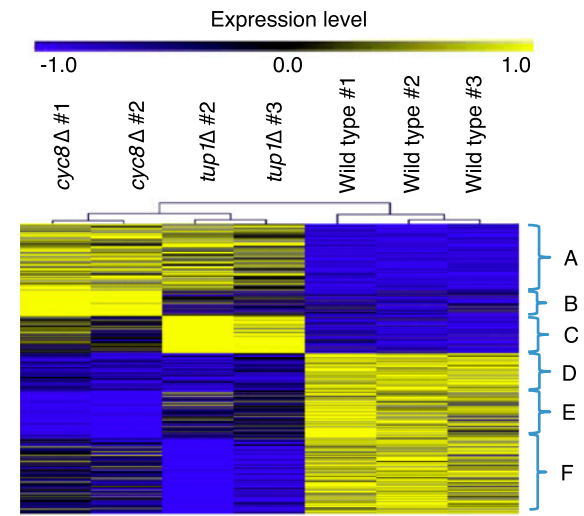

$\left.\begin{array}{r}6000 \\ 4000 \\ 2000 \\ 0\end{array}\right]$

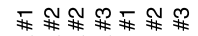

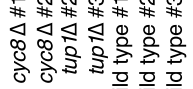
은 은 은

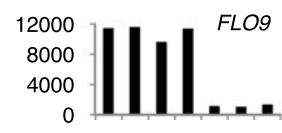

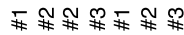

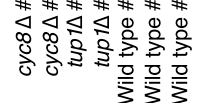

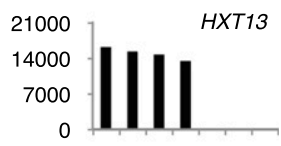

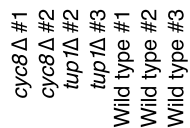

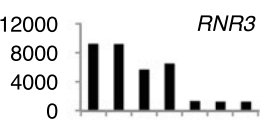

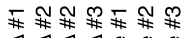

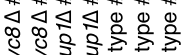

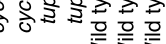

Figure 3. Cyc8 and Tup1 show common and individual global effects on transcriptional regulation. $(A)$ Venn diagram showing the overlap of regulated genes ( $Q$-value $<0.05$; fold change $>1.5$ ) in cyc8s or tup $1 \Delta$ strain relative to wild-type strain. The overlap $P$-value is estimated based on a Fisher's exact test. $(B)$ A heat map to show gene expression level in each sample. Each row represents a differentially expressed gene in the $c y c 8 \Delta$ or tup $1 \Delta$ strain relative to the wild-type strain, and each column represents a sample. Samples were hierarchically clustered, and the expression values for each gene from all seven samples were scaled to a range between -1 and 1. Nucleosomes were grouped and labeled based on the Venn diagrams in $A$. (C) Histograms showing expression levels of four known Cyc8Tup1 target genes in cyc $8 \Delta$, tup $1 \Delta$, and wild-type strains.

genes (Fig. 3A), confirming that the primary common function of Cyc8 and Tup1 is to regulate gene expression negatively. This observation becomes more obvious when we increased the stringency 
of the cutoffs to define regulated genes. For example, based on a $Q$-value cutoff of 0.01 and a fold change cutoff of 2, 185 genes are up-regulated in both $c y c 8 \Delta$ and tup1s strains, with an overlapping $P$-value of $1 \times 10^{-119}$, whereas only 33 common genes are down-regulated, with an overlapping $P$-value of $1 \times 10^{-36}$ (Supplemental Fig. 6). Functional enrichment analyses revealed that the up-regulated genes play major roles in transcriptional regulation and sugar trans-membrane transport activities (Table 2).

A few differences in global transcriptional effects of $C Y C 8$ and TUP1 loss were also observed. More genes were down-regulated in tup1s strains than in $c y c 8 \Delta$ strains (Fig. 3A); this trend was maintained even with an increase in the cutoff stringency used to define regulated genes (Supplemental Fig. 6). Indeed, genes classified in a "RNA polymerase activity" Ontology group are enriched in the set of genes that are down-regulated in tup $1 \Delta$ strains; however, this enrichment was not observed for genes down-regulated in $c y c 8 \Delta$ strains (Table 2).

\section{Cyc8 and Tup1 repress TATA-containing genes by nucleosome reorganization}

The Cyc8-Tup1 complex may preferentially regulate genes containing TATA-boxes on promoters. Our analyses revealed a strong preference of Cyc8 and Tup1 binding to promoters of TATA-containing genes based on public Cyc8 and Tup1 ChIP-chip data (Fig. 4A; Venters et al. 2011). By ranking genes based on the intensity of Cyc 8 or Tup 1 binding at promoters, we observed that $25 \%$ of the top 1000 genes bound by Cyc 8 and $22 \%$ of the top 1000 genes bound by Tup 1 have TATA-containing promoters. The percentage of TATA-containing promoters decreased in lower-ranking Cyc8or Tup1-bound genes and is at least 5 percentage points lower in the middle or bottom 1000 genes. We also observed a strong correlation between the frequency of TATA-containing genes and the significance of expression up-regulation in $c y c 8 \Delta$ or tup $1 \Delta$ strains relative to wild-type cells; however, this correlation was not observed for down-regulated genes (Fig. 4B). For genes that were upregulated, the correlation coefficients reach 0.92 in $c y c 8 \Delta$ strains and 0.85 in tup1s strains, respectively; however, the correlation coefficients are only 0.03 and 0.12 for down-regulated genes.

Table 2. Biological features associated with differential genes in cyc8s or tup $1 \Delta$

\begin{tabular}{|c|c|c|c|c|c|}
\hline \multirow[b]{2}{*}{$\begin{array}{l}\text { Feature } \\
\text { category }\end{array}$} & \multirow[b]{2}{*}{$\begin{array}{l}\text { Feature } \\
\text { name }\end{array}$} & \multicolumn{2}{|c|}{$\begin{array}{c}\text { cyc8s } \\
\text { expression }\end{array}$} & \multicolumn{2}{|c|}{$\begin{array}{c}\text { tup } 1 \Delta \\
\text { expression }\end{array}$} \\
\hline & & Up & Down & Up & Down \\
\hline \multirow[t]{8}{*}{ Major function (GO terms) } & Transcription factor activity & $\mathrm{Y}$ & & $\mathrm{Y}$ & \\
\hline & Sequence-specific DNA binding & $\mathrm{Y}$ & & $\mathrm{Y}$ & \\
\hline & $\begin{array}{l}\text { Sugar transmembrane transporter } \\
\text { activity }\end{array}$ & Y & & Y & \\
\hline & Symporter activity & $\mathrm{Y}$ & & $\mathrm{Y}$ & \\
\hline & RNA polymerase activity & & & & $\mathrm{Y}$ \\
\hline & RNA binding & & & & $\mathrm{Y}$ \\
\hline & Nucleotide binding & & & & $\mathrm{Y}$ \\
\hline & $\begin{array}{l}\text { ATP-dependent RNA helicase } \\
\text { activity }\end{array}$ & & & & Y \\
\hline \multirow{3}{*}{$\begin{array}{l}\text { Nucleosome change } \\
\text { (TSS }-500 \text { to } 500 \mathrm{bp} \text { ) }\end{array}$} & Nucleosome gaining & $\mathrm{Y}$ & & $\mathrm{Y}$ & \\
\hline & Nucleosome loss & $\mathrm{Y}$ & & $\mathrm{Y}$ & \\
\hline & Nucleosome shift & $\mathrm{Y}$ & & $\mathrm{Y}$ & \\
\hline \multirow{2}{*}{ Cyc8-Tup1 binding } & Cyc8 binding & $\mathrm{Y}$ & & $\mathrm{Y}$ & \\
\hline & Tup1 binding & $\mathrm{Y}$ & & $\mathrm{Y}$ & \\
\hline
\end{tabular}

$\mathrm{Y}$ is assigned when $P<1 \times 10^{-5}$ or the difference of $P$ between up-regulated and down-regulated reaches 1000-fold, or the $Q$-value $<0.05$ for $\mathrm{GO}$ terms.
We then asked whether the Cyc8-Tup1 complex was physically associated with the promoters of their putative gene targets. Genes up-regulated in $c y c 8 \Delta$ strains significantly overlapped with genes bound by Cyc 8 or Tup 1 in wild-type cells. Similar results were observed for genes up-regulated in tup1s strains (Table 2), suggesting that Cyc8 and Tup1 bind to promoters of these genes and repress their transcription. However, no significant overlap was observed between Cyc8-Tup1-bound genes and genes that are down-regulated in the $c y c 8 \Delta$ or tup $1 \Delta$ strains, indicating that these genes are not direct targets of Cyc8-Tup1, and that the downregulation in tup $1 \Delta$ or $c y c 8 \Delta$ strains is likely a secondary effect of CYC8 or TUP1 loss. In addition, we observed both gain and loss of nucleosomes on promoters of up-regulated genes; in contrast, down-regulated genes were not significantly overlapped with genes with nucleosome changes on promoters (Table 2 ). Collectively, these results suggest that Cyc8-Tup1 target genes tend to be TATA-containing genes up-regulated in $c y c 8 \Delta$ and tup $1 \Delta$ strains along with changes in nucleosome positioning, and genes that were down-regulated in these strains, however, are likely secondary or nonspecific targets.

To further investigate the direct effect of Cyc8-Tup1 on chromatin structures, we defined 281 Cyc8-Tup1 direct target genes that show Cyc8 or Tup1 binding in wild-type cells and expression increase upon CYC 8 or TUP1 loss. We then plotted nucleosome occupancy change flanking TSS of the target genes. For comparison, the same plot was also done for all yeast genes (Fig. 4C). The effects of Cyc8-Tup1 loss on direct target genes showed the same trend with their global effects on all yeast genes, whereas the effect on target genes is clearly stronger than the global effects. More severe nucleosome loss was observed on both promoters and gene bodies of the target genes relative to all yeast genes, whereas nucleosome gain was observed preferentially on gene bodies.

\section{A typical example for the functional mechanisms of the Cyc8-Tupl complex}

Although the chromatin alterations in tup $1 \Delta$ and $c y c 8 \Delta$ strains on some Cyc8-Tup1 target genes appear to be pure nucleosome loss on promoters, e.g., for the genes HXT13 and FLO9 (Fig. 2A), a more general trend revealed by statistical analysis is extensive nucleosome rearrangement including both nucleosome gain and loss between the promoter and the $5^{\prime}$ end of the gene body (Figs. 2D, 4C), as was observed in the cases of STE2 and RNR3 (Fig. 2A). The promoter region of the HXT8 gene represents a typical target region of the Cyc8-Tup1 complex (Fig. 5). A TATA-box is located 85 bp upstream of the start codon of HXT8 (Fig. 5A), the P nucleosome is located immediately upstream of the TATA-box of HXT8 in wildtype cells (Fig. 5B), and Cyc8 and Tup1 binding was observed on the promoter (Fig. 5C). The P nucleosome disappears in both $c y c 8 \Delta$ and tup1s strains; interestingly, the -1 and +1 nucleosomes flanking the P nucleosomes are shifted in these strains. As a result, all the neighboring nucleosomes in this region appear to be shifted by half of a nucleosome size. The loss of the $\mathrm{P}$ nucleosome in the ab- 
sence of $C Y C 8$ or TUP1 would allow transcription factors to bind the promoter sequence, which could then facilitate transcriptional activation, as can be proved by the derepression of $H X T 8$ in $c y c 8 \Delta$ and tup1s cells (Fig. 5D). The gene IMA5 that is located $797 \mathrm{bp}$ upstream of HXT8 on the reverse strand shares a common intergenic region with $H X T 8$, and a TATA-box is located 87 bp upstream of this gene; interestingly, similar trends in Cyc8-Tup1 binding, nucleosome rearrangement, and expression change are also observed for this gene (Fig. 5).

A

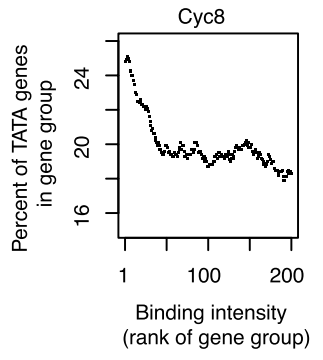

B
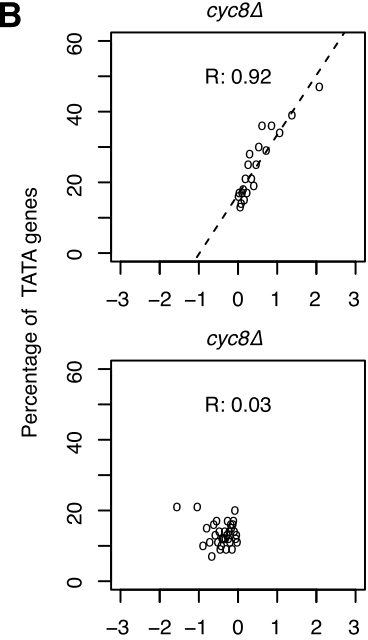

Log2 expression fold change
C

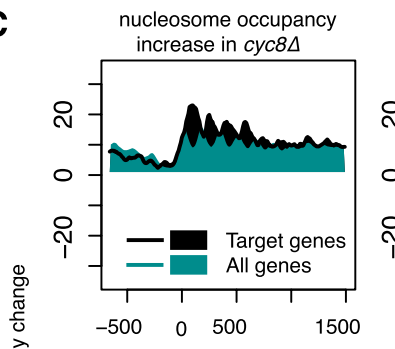

nucleosome occupancy increase in tup1 $\triangle$

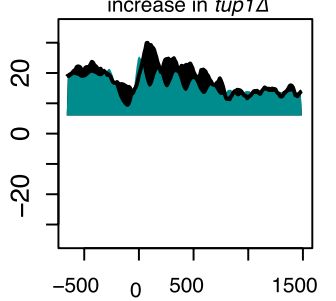

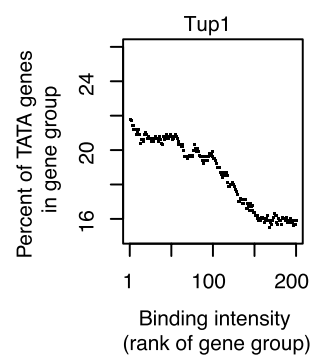
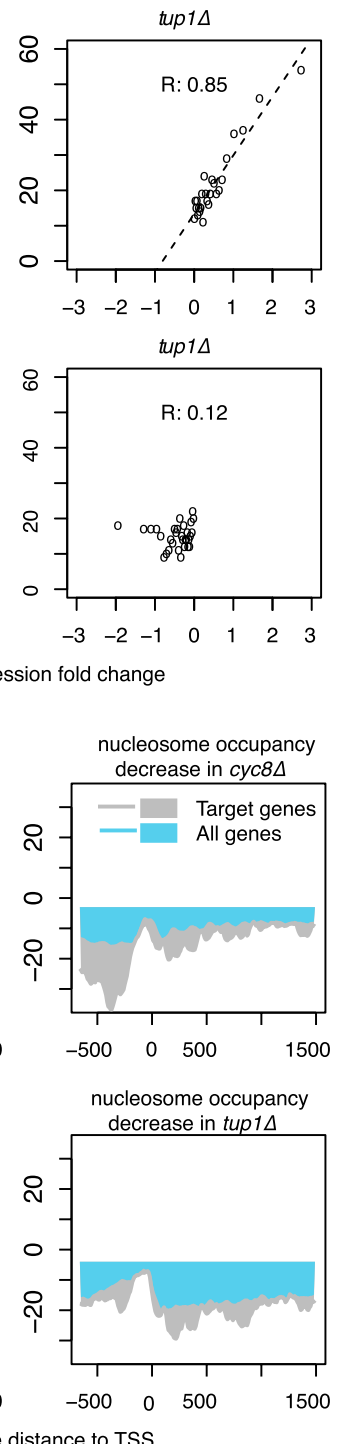

\section{Discussion}

Previous investigations of Cyc8-Tup1 function in gene expression regulation were limited in focus to single or several genes, providing locus-specific details for the functional mechanisms of this corepressor (Cooper et al. 1994; Ozcan and Johnston 1995; Fleming and Pennings 2001; Li and Reese 2001; Davie et al. 2002). Until now, the mechanisms underlying Cyc8-Tup1-mediated repression of only a handful of genes had been explored. The nucleosome occupancy profiling in this work provides an opportunity to look at the Cyc8-Tup1 effect at single-nucleotide resolution. By whole-genome-scale comparative analysis of nu-

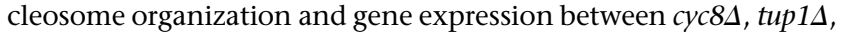
and wild-type cells, we are now able to summarize some general features of Cyc8-Tup1 function in a statistical way. Public genomic data, such as the ChIP-chip profiling of Cyc8-Tup1 binding sites (Venters et al. 2011) and TATA-containing gene set (Basehoar et al. 2004), also provide robust resources for discerning general mechanisms of the Cyc8-Tup1 corepressor.

Multiple maps of genome-wide nucleosome locations in Saccharomyces cerevisiae are now available (Jiang and Pugh 2009). These studies indicate that nucleosome locations play a large role in transcriptional regulation of gene expression by allowing and/or blocking the access of transcriptional modulators. Similarly, our identification of the Cyc8-Tup1-dependent P nucleosome in the previously characterized nucleosome-free region (NFR) suggests a new mode of regulation by variation in nucleosome position and occupancy. The NFR is highly enriched for binding sites, such as the TATA-box, and is recognized by specific transcription factors (Xiao et al. 1998; Sun et al. 2009) that typically bind close to the location of transcriptional initiation sites. Therefore, factors that positively and negatively regulate NFR accessibility may ultimately affect transcriptional activation.

Although most high-throughput nucleosome positioning investigations reveal an NFR region at the location immediately upstream of TSS between the $-111 \mathrm{bp}$ and $-5 \mathrm{bp}$, some reports indicate that TSS proximal regions for some genes can also be occupied by promoter nucleosomes. The P nucleosomes we observed are different from these previously reported promoter nucleosomes. For example, a recent work revealed a set of promoter nucleosomes that tend to retain high occupancy. These nucleosomes are fuzzy and are evenly distributed across the entire promoter region ( $-400 \mathrm{bp}$ to $0 \mathrm{bp}$ relative to the TSS) but not necessarily in

Figure 4. The Cyc8-Tup1 complex regulates TATA-containing genes via nucleosome organization. (A) Frequency of TATA-containing genes plotted as a function of $\mathrm{Cyc} 8$ or Tup1 binding intensity. We ranked the genes by Cyc 8 or Tup1 binding intensity from highest to lowest, and use 1000-gene sliding windows in 10-gene increments down each rank to produce overlapping groups. Then the percentage of TATA-containing genes in each group was calculated. (B) Frequency of TATA-containing genes plotted as function of fold expression change in the $c y c 8 \Delta$ or the tup $1 \Delta$ strain relative to the wild-type strain. Genes were ranked based on the expression fold change and grouped into 100-gene bins with no overlap between any two bins. The fraction of the TATA-containing genes in each bin was plotted against the corresponding average gene expression fold change. Correlation coefficients were calculated based on the Spearman method. Up-regulated genes (top panels) and down-regulated genes (bottom panels) were analyzed separately. (C) Nucleosome occupancy change plotted as function of distance to transcription start sites of either all yeast genes or Cyc8-Tup1 target genes, as defined by Cyc8Tup1 binding and expression change upon loss of these factors. Nucleosome occupancy increases (left panels) and occupancy decreases (right panels) were analyzed separately.

\section{Genome Research}


A

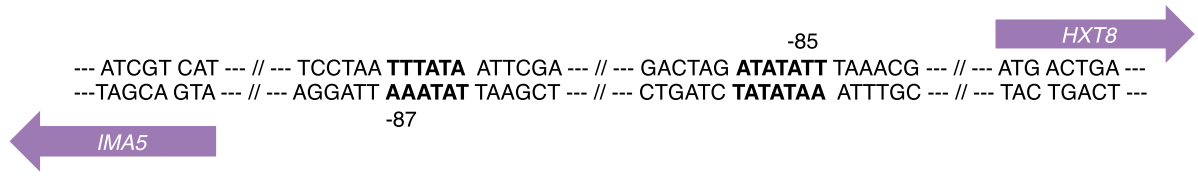

B
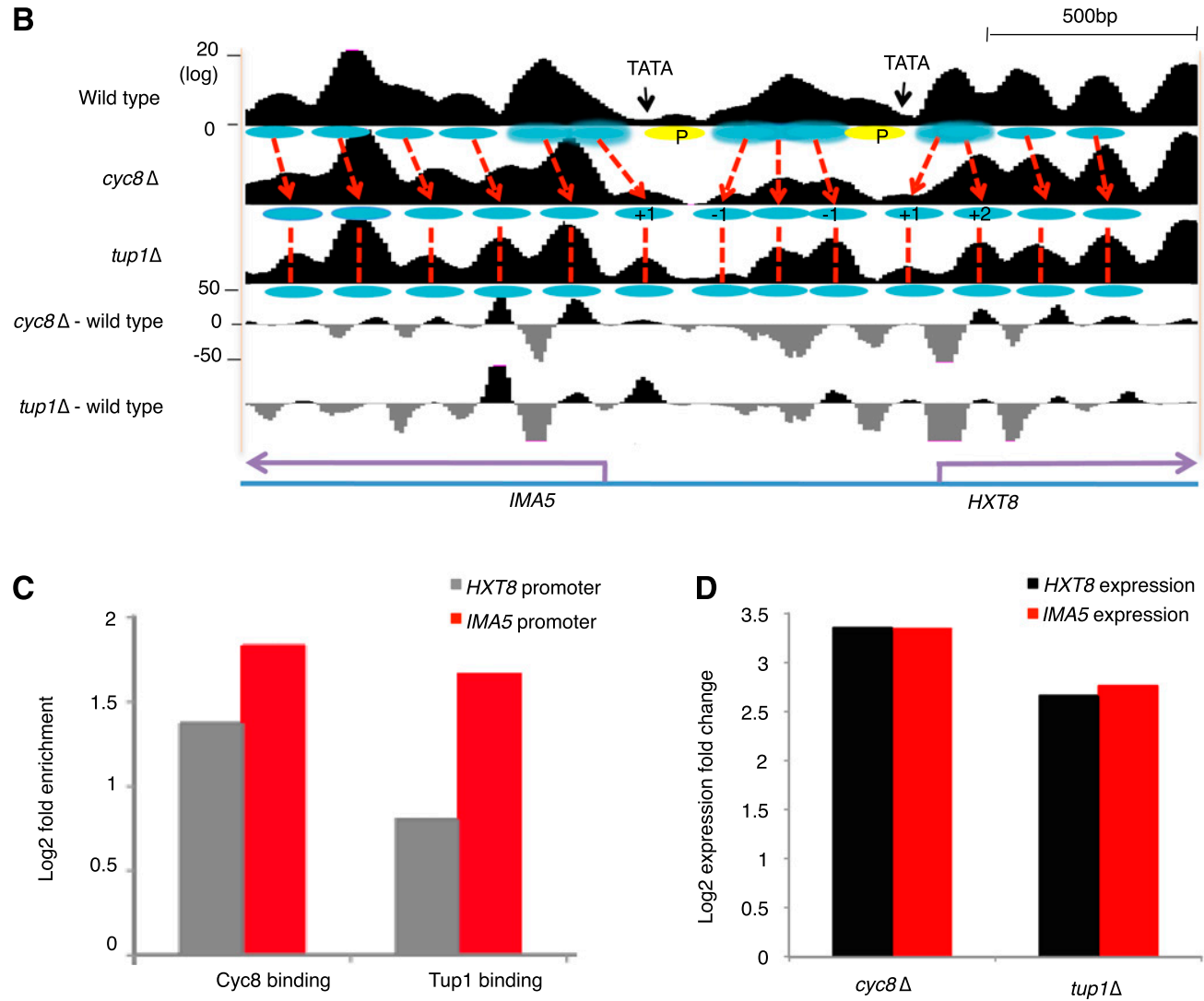

Figure 5. A typical organization of nucleosomes by the Cyc8-Tup1 corepressor complex. (A) The intergenic region between $H X T 8$ and IMA5 contains two TATA boxes (bold black) located 85 and 87 bp upstream of the start codons of HXT8 and IMA5, respectively. (B) Nucleosome occupancy and occupancy change across the promoter regions between HXT8 and IMA5. (Ovals) Canonical nucleosomes (skyblue) or the "P nucleosome" (yellow) defined in this study. Genes (purple arrows) are labeled below. Each of the top three tracks represents nucleosome occupancy in each sample. The cyc84wild-type and tup $1 \Delta$-wild-type tracks show the net occupancy difference between the corresponding samples and were plotted to show nucleosome gain (black; positive) or nucleosome loss (gray; negative). (Red dashed lines) The shift of nucleosomes between cell types. (C) Cyc8 and Tup1 binding enrichment on the promoters of $H X T 8$ and IMAS based on public ChIP-chip data in wild-type strains. (D) Expression change of HXT8 and IMAS in $C y C 8 \Delta$ and tup1s strains based on microarray data.

the NFR (-111 bp to 5 bp) (Tirosh and Barkai 2008). As a result, the associated promoter does not have a robust nucleosome pattern composed of a well-positioned -1 nucleosome, an NFR, and a wellpositioned +1 nucleosome. The previously described promoter nucleosomes indicate high plasticity of gene expression but do not imply gene repression. In contrast, the $\mathrm{P}$ nucleosomes that we describe have low occupancy and are located in the canonical nucleosome-free regions (NFR) with well-positioned flanking -1 and +1 nucleosomes. Furthermore, the $\mathrm{P}$ nucleosomes are stabilized as part of the repressive chromatin architecture created by the Cyc8-Tup1 corepressor, and thus contribute to transcription repression.

Stabilization of the P nucleosome in canonical NFRs of TATAcontaining genes as we observe for the Cyc8-Tup1 corepressor may reflect a common mechanism for repression, because multiple factors appear to positively or negatively regulate the NFR. Our analysis based on public data sets indicate that at least eight of 25 chromatin-remodeling factors show stronger binding on TATAcontaining promoters relative to the promoters that contain no TATA-box (Supplemental Fig. 7). These factors include Rsc8 (Cairns et al. 1996), Nhp6a (Stillman 2010), Asf1 (Green et al. 2005), Swi3 (Sarnowski et al. 2002), Itc1 (Ruiz et al. 2003), and Pob3 (Brewster et al. 1998), as well as Cyc8 and Tup1 investigated in this study. Four factors appear to prefer to bind promoters that contain no TATA-box, including Aor1 (Krogan et al. 2003), Swr1 (Mizuguchi et al. 2004), Vps72 (Wu et al. 2005), and Swc1 (Krogan et al. 2003). Interestingly, all these factors are components of the Swr1 complex, which exchanges the histone H2A.Z for H2A on promoters. Previous investigations revealed that the Rsc complex can exclude nucleosomes in the NFR and increase its size, which results in the increase in accessibility of transcription factors that positively regulate the expression of Rsc target genes (Badis et al. 2008; 
Hartley and Madhani 2009). Only recently has a negative regulator of the NFR region been described. The ATP-dependent Isw2 remodeling complex can negatively regulate the size of the NFR, and hence accessibility of transcriptional activators, by sliding nucleosomes from the gene bodies and into the middle of the NFRs of many of its targets (Yadon et al. 2010; Sadeh and Allis 2011).

The functions of Cyc8-Tup1 have been linked to the Isw2 complex (Zhang and Reese 2004a,b; Rizzo et al. 2011). However, previous studies of Cyc8-Tup1-mediated regulation of nucleosome positions at target genes have not previously described the existence of the P nucleosome in the NFR. It is likely that this P nucleosome was not previously detected because of its low occupancy levels. Our data combined with previous studies suggest that the Isw2 complex may be used to mobilize nucleosomes into preferred positions for repression, and the Cyc8-Tup1 complex may function to specifically lock the P nucleosome over the TATA-box in order to block access to the TATA-binding protein and negatively regulate expression of gene targets.

\section{Methods}

\section{Yeast knockout strains}

Yeast strains were created by standard procedures by amplifying the knockout cassette from the corresponding strain in the Open Biosystems yeast knockout collection and transforming it into BY4742 strains. Transformants were selected by G418 resistance and confirmed by standard PCR techniques.

\section{Isolation of nucleosomal DNA}

To determine the differences in nucleosome positioning in yeast between wild-type (BY4742), cyc8s, and tup1 1 strains, yeast strains BY4742, YSC1021-550429, yMW130, yMW147, and yMW149 were grown to a final $\mathrm{OD}_{600 \mathrm{~nm}}$ of 0.8 in $200 \mathrm{~mL}$ of YEP containing $2 \%$ glucose. Cells were chemically cross-linked for $30 \mathrm{~min}$ by adding formaldehyde solution to a final concentration of $2 \%$. The cross-linking reaction was then quenched for $5 \mathrm{~min}$ following the addition of glycine to a final concentration of $125 \mathrm{mM}$. Cells were then collected and washed two times in $20 \mathrm{~mL}$ of $1 \times$ PBS. Next, each sample was resuspended in $3 \mathrm{~mL}$ of a $1 \mathrm{M}$ sorbitol, $50 \mathrm{mM}$ Tris$\mathrm{HCl}(\mathrm{pH}$ 7.4) solution with freshly added $10 \mathrm{mM} \beta$-mercaptoethanol. Then $0.25 \mathrm{mg} / \mathrm{mL}$ 100T Zymolase (ICN) was added to each sample, and samples were incubated for $30 \mathrm{~min}$ at $30^{\circ} \mathrm{C}$ while shaking. Next, samples were harvested and resuspended in $2 \mathrm{~mL}$ of a $1 \mathrm{M}$ sorbitol, $50 \mathrm{mM} \mathrm{NaCl}, 10 \mathrm{mM}$ Tris- $\mathrm{HCl}$ (pH 7.4), $5 \mathrm{mM} \mathrm{MgCl}$, $1 \mathrm{mM} \mathrm{CaCl}$, and $0.075 \%$ Nonidet-P-40 solution containing 100 units of RNase I (Roche) and freshly added $1 \mathrm{mM} \beta$-mercaptoethanol and $500 \mu \mathrm{M}$ spermidine. Samples were then aliquoted into 6 Eppendorf tubes containing $0,0.1 \mathrm{unit} / \mathrm{mL}, 0.2 \mathrm{unit} / \mathrm{mL}, 0.3$ unit $/ \mathrm{mL}, 0.4 \mathrm{unit} / \mathrm{mL}$, or $0.6 \mathrm{unit} / \mathrm{mL}$ of MNase (Sigma-Aldrich). Samples were incubated for $45 \mathrm{~min}$ at $37^{\circ} \mathrm{C}$ on a nutator. To terminate the MNase digestion, $75 \mu \mathrm{L}$ of a $5 \%$ SDS, $50 \mathrm{mM}$ EDTA solution was added to each sample. Next, $15 \mu \mathrm{L}$ of Proteinase $\mathrm{K}$ solution ( $20 \mathrm{mg} / \mathrm{mL}$; Sigma-Aldrich) was added to each sample, and samples were then incubated overnight at $65^{\circ} \mathrm{C}$. DNA was isolated from each sample by standard phenol:chloroform extraction techniques and resuspended in $30 \mu \mathrm{L}$ of DNA loading buffer and 2 units of RNase I (Roche). Samples were then subjected to electrophoresis through $2 \%$ agarose, and the DNA from each array of time points migrating near the 150-bp mark was excised, combined, and purified by using the QIAGEN gel extraction kit (QIAGEN) and then subjected to another round of phenol: chloroform extraction.

\section{Nucleosome occupancy calculation}

Short sequencing reads were mapped to the $S$. cerevisiae reference genome (version SGD/sacCer1, UCSC Oct 2003) by ELAND and then analyzed based on DANPOS (http://code.google.com/p/ danpos/) (Chen et al. 2012). The average size of the DNA fragments in each sample was estimated by cross-strand Pearson correlation. The $5^{\prime}$ end of each uniquely mapped and highquality read was shifted half a fragment size toward the $3^{\prime}$ end of the DNA and then extended $37 \mathrm{bp}$ in both directions. Nucleosome occupancy at each base pair was calculated as read coverage. After calculating occupancy for each sample, we performed quantile normalization for all nine samples (three biological replicates per strain). Reference occupancy data for each of the three strains were generated by calculating the average of the three replicates.

\section{Nucleosome calling}

Nucleosome positions were first called by using a sliding window of $40 \mathrm{bp}$ to identify a "bell"-shaped curve supported by at least five reads, with the occupancy summit in the middle of the sliding window. Neighboring "bell"-shaped curves that were $<110 \mathrm{bp}$ apart were merged as one curve. Each nucleosome was then determined by the summit and neighboring edges of the "bell"-shaped curve. The edges were determined by searching for the lowest flanking occupancy valleys. We set the requirements such that the nucleosome edges were at least $40 \mathrm{bp}$ but no more than 110 bp away from the summit. Each nucleosome call was counted once in plotting average nucleosome count flanking TSS, and the position of each count was assigned to the summit position.

\section{Data reproducibility analysis}

We generated reference occupancy data by taking the average of all nine samples, in which a total of 66,167 reference nucleosomes were identified. For each reference nucleosome, the occupancy summit value was retrieved from each of the nine samples to construct a $66,167 \times 9$ data matrix for the reproducibility analysis with the following three metrics: (1) pairwise Pearson correlation of occupancy between replicates; (2) Fisher's exact $P$-value for the overlap of high-occupancy nucleosomes between replicates (highoccupancy nucleosomes of each replicate were identified as those whose occupancy values were higher than the genome average); and (3) hierarchical clustering of all nine samples based on Euclidean distance of the top 5\% of nucleosomes with a high standard deviation (SD).

\section{Detecting nucleosome changes}

With the current genome coverage, tag distribution along the genome could be modeled by a Poisson distribution, with one parameter $\lambda$ for both the mean and the variance of the distribution. To estimate the significance of observed nucleosome occupancy at each base pair in a treatment sample, we calculated the $P$-value based on Poisson distribution, with $\lambda$ defined by the nucleosome occupancy at the same base pair in a control sample. To compensate for the potential experimental variations between the biological replicates, three replicates of each strain were merged, and then nucleosome changes were identified between strains. Next, Poisson-based $P$-values at each base pair were transformed to a score as $-\log _{10}\left(P\right.$-value) (e.g., a $P$-value of $1 \times 10^{-5}$ is transformed to 5). Differential nucleosome peaks were called from the score data based on the same method used for nucleosome calling.

\section{Genome Research}




\section{Microarray and sequencing}

All information related to microarray, sequencing, and PCR experiments are provided in the Supplemental material.

\section{Data access}

All data have been submitted to the NCBI Gene Expression Omnibus (GEO) (http://www.ncbi.nlm.nih.gov/geo/) under accession number GSE37467.

\section{Acknowledgments}

We thank Boyko Atanassov, Marek Napierela, and Kadir Akdemir (UT MD Anderson Cancer Center) for fruitful discussions. This work was supported by the National Institute of Child Health and Human Development Training Grant to M.A.W. in "Differentiation and Development" (2 T32 HD07325) and grants from the NIH (R01GM51189) and the MDACC Senior Research Trust to S.Y.R.D. The microarray work described was supported by the Vermont Genetics Network through Grant Number 2P20RR016462 from the INBRE Program of the National Center for Research Resources (NCRR), a component of the National Institutes of Health (NIH). Its contents are solely the responsibility of the authors and do not necessarily represent the official views of NCRR or NIH.

Authors' contributions: W.L. and S.Y.R.D. conceived the project; W.L., M.A.W., and S.Y.R.D. designed the experiments and also analyzed the data; M.A.W. performed the experiments; and K.C. built up the data analysis pipeline, analyzed the data, and plotted the figures. C.H. provided technical help with Rt-PCR experiments; A.W. performed the microarray experiments; S.L. and Y.L. provided initial nucleosome mapping feasibility analyses; and M.A.W., K.C., W.L., and S.Y.R.D. wrote the manuscript.

\section{References}

Badis G, Chan ET, van Bakel H, Pena-Castillo L, Tillo D, Tsui K, Carlson CD, Gossett AJ, Hasinoff MJ, Warren CL, et al. 2008. A library of yeast transcription factor motifs reveals a widespread function for Rsc3 in targeting nucleosome exclusion at promoters. Mol Cell 32: 878-887.

Basehoar AD, Zanton SJ, Pugh BF. 2004. Identification and distinct regulation of yeast TATA box-containing genes. Cell 116: 699-709.

Brewster NK, Johnston GC, Singer RA. 1998. Characterization of the CP complex, an abundant dimer of Cdc68 and Pob3 proteins that regulates yeast transcriptional activation and chromatin repression. J Biol Chem 273: 21972-21979.

Burke LJ, Baniahmad A. 2000. Co-repressors 2000. FASEB J 14: 1876-1888.

Buscarlet M, Stifani S. 2007. The 'Marx' of Groucho on development and disease. Trends Cell Biol 17: 353-361.

Cairns BR, Lorch Y, Li Y, Zhang M, Lacomis L, Erdjument-Bromage $\mathrm{H}_{\text {, }}$ Tempst P, Du J, Laurent B, Kornberg RD. 1996. RSC, an essential, abundant chromatin-remodeling complex. Cell 87: 1249-1260.

Chen K, Xi Y, Pan X, Li Z, Kaestner K, Tyler J, Dent S, He X, Li W. 2012. DANPOS: Dynamic analysis of nucleosome position and occupancy by sequencing. Genome Res (this issue). doi: 10.1101/gr.142067.112.

Chinnadurai G. 2003. CtBP family proteins: More than transcriptional corepressors. Bioessays 25: 9-12.

Conlan RS, Gounalaki N, Hatzis P, Tzamarias D. 1999. The Tup1-Cyc8 protein complex can shift from a transcriptional co-repressor to a transcriptional co-activator. J Biol Chem 274: 205-210.

Cooper JP, Roth SY, Simpson RT. 1994. The global transcriptional regulators, SSN6 and TUP1, play distinct roles in the establishment of a repressive chromatin structure. Genes Dev 8: 1400-1410.

Davie JK, Trumbly RJ, Dent SY. 2002. Histone-dependent association of Tup1-Ssn6 with repressed genes in vivo. Mol Cell Biol 22: 693-703.

Davie JK, Edmondson DG, Coco CB, Dent SY. 2003. Tup1-Ssn6 interacts with multiple class I histone deacetylases in vivo. J Biol Chem 278: 50158-50162.

Ducker CE, Simpson RT. 2000. The organized chromatin domain of the repressed yeast a cell-specific gene STE6 contains two molecules of the corepressor Tup1p per nucleosome. EMBO J 19: 400-409.
Fleming AB, Pennings S. 2001. Antagonistic remodelling by Swi-Snf and Tup1-Ssn 6 of an extensive chromatin region forms the background for FLO1 gene regulation. EMBO J 20: 5219-5231.

Fleming AB, Pennings S. 2007. Tup1-Ssn6 and Swi-Snf remodelling activities influence long-range chromatin organization upstream of the yeast SUC2 gene. Nucleic Acids Res 35: 5520-5531.

Green EM, Antczak AJ, Bailey AO, Franco AA, Wu KJ, Yates JR, Kaufman PD. 2005. Replication-independent histone deposition by the HIR complex and Asf1. Curr Biol 15: 2044-2049.

Hartley PD, Madhani HD. 2009. Mechanisms that specify promoter nucleosome location and identity. Cell 137: 445-458.

Heinzel T, Lavinsky RM, Mullen TM, Soderstrom M, Laherty CD, Torchia J, Yang WM, Brard G, Ngo SD, Davie JR, et al. 1997. A complex containing $\mathrm{N}-\mathrm{CoR}, \mathrm{mSin} 3$ and histone deacetylase mediates transcriptional repression. Nature 387: 43-48.

Jiang C, Pugh BF. 2009. A compiled and systematic reference map of nucleosome positions across the Saccharomyces cerevisiae genome. Genome Biol 10: R109. doi: 10.1186/gb-2009-10-10-r109.

Keleher CA, Redd MJ, Schultz J, Carlson M, Johnson AD. 1992. Ssn6-Tup1 is a general repressor of transcription in yeast. Cell 68: 709-719.

Kim J-H, Polish J, Johnston M. 2003. Specificity and regulation of DNA binding by the yeast glucose transporter gene repressor Rgt1. Mol Cell Biol 23: 5208-5216.

Krogan NJ, Keogh M-C, Datta N, Sawa C, Ryan OW, Ding H, Haw RA, Pootoolal J, Tong A, Canadien V, et al. 2003. A Snf2 family ATPase complex required for recruitment of the histone H2A variant Htz1. Mol Cell 12: $1565-1576$.

Li B, Reese JC. 2001. Ssn6-Tup1 regulates RNR3 by positioning nucleosomes and affecting the chromatin structure at the upstream repression sequence. J Biol Chem 276: 33788-33797.

Li J, Wang J, Nawaz Z, Liu JM, Qin J, Wong J. 2000. Both corepressor proteins SMRT and N-CoR exist in large protein complexes containing HDAC3. EMBO J 19: 4342-4350.

Mizuguchi G, Shen X, Landry J, Wu W-H, Sen S, Wu C. 2004. ATP-driven exchange of histone $\mathrm{H} 2 \mathrm{AZ}$ variant catalyzed by SWR1 chromatin remodeling complex. Science 303: 343-348.

Nagy L, Kao HY, Chakravarti D, Lin RJ, Hassig CA, Ayer DE, Schreiber SL, Evans RM. 1997. Nuclear receptor repression mediated by a complex containing SMRT, mSin3A, and histone deacetylase. Cell 89: 373380.

Ozcan S, Johnston M. 1995. Three different regulatory mechanisms enable yeast hexose transporter (HXT) genes to be induced by different levels of glucose. Mol Cell Biol 15: 1564-1572.

Papamichos-Chronakis M, Petrakis T, Ktistaki E, Topalidou I, Tzamarias D. 2002. Cti6, a PHD domain protein, bridges the Cyc8-Tup1 corepressor and the SAGA coactivator to overcome repression at GAL1. Mol Cell 9: 1297-1305.

Payankaulam S, Li LM, Arnosti DN. 2010. Transcriptional repression: Conserved and evolved features. Curr Biol 20: R764-R771.

Perissi V, Jepsen K, Glass CK, Rosenfeld MG. 2010. Deconstructing repression: Evolving models of co-repressor action. Nat Rev Genet 11: 109-123.

Rizzo JM, Mieczkowski PA, Buck MJ. 2011. Tup1 stabilizes promoter nucleosome positioning and occupancy at transcriptionally plastic genes. Nucleic Acids Res 39: 8803-8819.

Rosell M, Jones MC, Parker MG. 2011. Role of nuclear receptor corepressor RIP140 in metabolic syndrome. Biochim Biophys Acta 1812: 919-928.

Roth SY, Dean A, Simpson RT. 1990. Yeast $\alpha 2$ repressor positions nucleosomes in TRP1/ARS1 chromatin. Mol Cell Biol 10: 2247-2260.

Ruiz C, Escribano V, Morgado E, Molina M, Mazón MJ. 2003. Cell-typedependent repression of yeast a-specific genes requires Itc1p, a subunit of the Isw2p-Itc1p chromatin remodelling complex. Microbiology 149: 341-351.

Sadeh R, Allis CD. 2011. Genome-wide "re"-modeling of nucleosome positions. Cell 147: 263-266.

Sarnowski TJ, Swiezewski S, Pawlikowska K, Kaczanowski S, Jerzmanowski A. 2002. AtSWI3B, an Arabidopsis homolog of SWI3, a core subunit of yeast Swi/Snf chromatin remodeling complex, interacts with FCA, a regulator of flowering time. Nucleic Acids Res 30: 3412-3421.

Shi Y, Sawada J, Sui G, Affar el B, Whetstine JR, Lan F, Ogawa H, Luke MP, Nakatani Y. 2003. Coordinated histone modifications mediated by a CtBP co-repressor complex. Nature 422: 735-738.

Silverstein RA, Ekwall K. 2005. Sin3: A flexible regulator of global gene expression and genome stability. Curr Genet 47: 1-17.

Smith RL, Johnson AD. 2000. Turning genes off by Ssn6-Tup1: A conserved system of transcriptional repression in eukaryotes. Trends Biochem Sci 25: 325-330.

Stillman DJ. 2010. Nhp6: A small but powerful effector of chromatin structure in Saccharomyces cerevisiae. Biochim Biophys Acta 1799: 175180. 
Chen et al.

Sun W, Xie W, Xu F, Grunstein M, Li KC. 2009. Dissecting nucleosome free regions by a segmental semi-Markov model. PLOS ONE 4: e4721. doi: 10.1371/journal.pone.0004721.

Tirosh I, Barkai N. 2008. Two strategies for gene regulation by promoter nucleosomes. Genome Res 18: 1084-1091.

Venters BJ, Wachi S, Mavrich TN, Andersen BE, Jena P, Sinnamon AJ, Jain P, Rolleri NS, Jiang C, Hemeryck-Walsh C, et al. 2011. A comprehensive genomic binding map of gene and chromatin regulatory proteins in Saccharomyces. Mol Cell 41: 480-492.

Watson AD, Edmondson DG, Bone JR, Mukai Y, Yu Y, Du W, Stillman DJ, Roth SY. 2000. Ssn6-Tup1 interacts with class I histone deacetylases required for repression. Genes Dev 14: 2737-2744.

Wu W-H, Alami S, Luk E, Wu C-H, Sen S, Mizuguchi G, Wei D, Wu C. 2005. Swc2 is a widely conserved H2AZ-binding module essential for ATP-dependent histone exchange. Nat Struct Mol Biol 12: 10641071.

Xi Y, Yao J, Chen R, Li W, He X. 2011. Nucleosome fragility reveals novel functional states of chromatin and poises genes for activation. Genome Res 21: 718-724.
Xiao G, White D, Bargonetti J. 1998. p53 binds to a constitutively nucleosome free region of the $m d m 2$ gene. Oncogene 16: 1171-1181.

Yadon AN, Van de Mark D, Basom R, Delrow J, Whitehouse I, Tsukiyama T. 2010. Chromatin remodeling around nucleosome-free regions leads to repression of noncoding RNA transcription. Mol Cell Biol 30: 51105122.

Zamir I, Zhang J, Lazar MA. 1997. Stoichiometric and steric principles governing repression by nuclear hormone receptors. Genes Dev 11: 835846.

Zhang Z, Reese JC. 2004a. Redundant mechanisms are used by Ssn6-Tup1 in repressing chromosomal gene transcription in Saccharomyces cerevisiae. J Biol Chem 279: 39240-39250.

Zhang Z, Reese JC. 2004b. Ssn6-Tup1 requires the ISW2 complex to position nucleosomes in Saccharomyces cerevisiae. EMBO J 23: 2246-2257.

Received April 20, 2012; accepted in revised form October 11, 2012. 


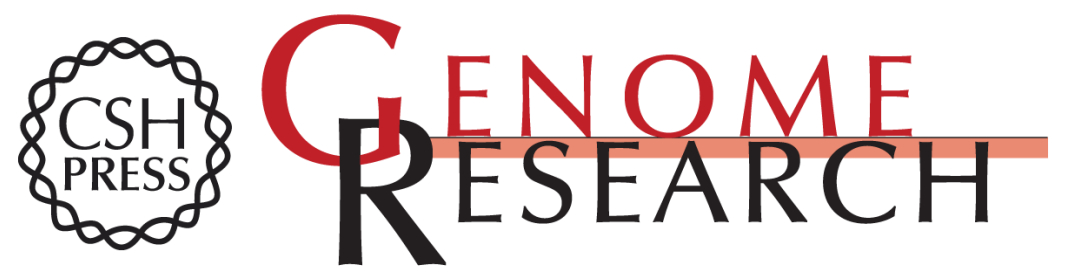

\section{Stabilization of the promoter nucleosomes in nucleosome-free regions by the yeast Cyc8-Tup1 corepressor}

Kaifu Chen, Marenda A. Wilson, Calley Hirsch, et al.

Genome Res. 2013 23: 312-322 originally published online November 2, 2012

Access the most recent version at doi:10.1101/gr.141952.112

Supplemental Material

References

Creative

Commons

License

Email Alerting

Service
http://genome.cshlp.org/content/suppl/2012/11/16/gr.141952.112.DC1

This article cites 50 articles, 20 of which can be accessed free at: http://genome.cshlp.org/content/23/2/312.full.html\#ref-list-1

This article is distributed exclusively by Cold Spring Harbor Laboratory Press for the first six months after the full-issue publication date (see

$\mathrm{http}: / / g$ enome.cshlp.org/site/misc/terms.xhtml). After six months, it is available under a Creative Commons License (Attribution-NonCommercial 3.0 Unported License), as described at http://creativecommons.org/licenses/by-nc/3.0/.

Receive free email alerts when new articles cite this article - sign up in the box at the top right corner of the article or click here.

\section{Affordable, Accurate Sequencing.}

To subscribe to Genome Research go to:

https://genome.cshlp.org/subscriptions 